\title{
Effects of study time and meaningfulness on environmental context-dependent recognition
}

\author{
Takeo Isarida • Toshiko K. Isarida • Tetsuya Sakai
}

Published online: 24 July 2012

(C) Psychonomic Society, Inc. 2012

\begin{abstract}
In two experiments, we examined whether the size of place-context-dependent recognition decreased with study time and with the meaningfulness of the to-beremembered materials. A group of 80 undergraduates intentionally studied a list of words in a short (1.5 s per item) or a long ( $4.0 \mathrm{~s}$ per item) study-time condition (Exp. 1). Another 40 undergraduates studied lists consisting of words and nonwords in the long-study-time condition (Exp. 2). After a short retention interval, recognition for the targets was tested in the same or in a different context. Context was manipulated by means of the combination of place, subsidiary task, and experimenter. Significant context-dependent recognition discrimination was found for words in the shortstudy-time condition (Exp. 1), but not in the long-study-time condition (Exps. 1 and 2). Significant effects were found as well for nonwords, even in the long-study-time condition (Exp. 2). These results are explained well by an outshining account: that is, by principles of outshining and encoding specificity.
\end{abstract}

Keywords Context-dependent effects · Environmental context $\cdot$ Recognition $\cdot$ Study time $\cdot$ Meaningfulness · Outshining principle

The encoding specificity principle (Tulving \& Thomson, 1973) and episode-defining contexts (Murnane, Phelps, \& Malmberg, 1999) have played important roles in accounting

T. Isarida $(\bowtie) \cdot$ T. Sakai

Faculty of Informatics, Shizuoka University,

3-5-1 Johoku,

Hamamatsu 432-8011, Japan

e-mail: isarida@inf.shizuoka.ac.jp

T. K. Isarida

Shizuoka Prefectural University,

Shizuoka, Japan for the remembering of episodes. An episodic-memory trace consists of focal information (the salient part of the episode) and context (the other parts of the episode). The context is encoded with the focal information into an episodicmemory trace and is used as a retrieval cue at the time of remembering. Because episodic memory is assumed to be encoded spatiotemporally (Tulving, 1972, 1983), the encoding specificity principle has been examined by manipulating a variety of places, such as on land versus underwater (e.g., Godden \& Baddeley, 1975), in a small room versus a wide garden (e.g., Bjork \& Richardson-Klavehn, 1989; Eich, 1995), in rooms with different features and objects (e.g., Smith, 1979; Smith, Glenberg, \& Bjork, 1978), and so forth (see Smith, 1988). Although these spatial contexts are usually called "environmental contexts," in the present study we refer to the spatial contexts manipulated by means of a variety of places as "place contexts," in order to distinguish the place context from other types of spatial context manipulated by means of a variety of visual features on a computer screen. These other visual features include the simple visual context, such as combinations of foreground color, background color, and item location (e.g., Murnane \& Phelps, 1993, 1994, 1995; Murnane et al., 1999); a background drawing (Murnane et al., 1999) or background photograph (Gruppuso, Lindsay, \& Masson, 2007; Hockley, 2008); and background color (e.g., Isarida, Isarida, \& Okamoto, 2005; Rutherford, 2004).

Place-context-dependent effects have been consistently found for recall (e.g., Godden \& Baddeley, 1975; Isarida \& Isarida, 2004; Smith, 1988; Smith \& Vela, 2001). Such effects in recall have been conceptualized as part of a body of evidence supporting the encoding specificity principle, because these effects are clearly explained by this principle.

In contrast, the evidence for place-context-dependent effects in recognition has been equivocal, especially with meaningful words. Most researchers once accepted the 
notion that place-context-dependent effects appear in recall but not in recognition (Baddeley, 1982; Godden \& Baddeley, 1975, 1980; Smith et al., 1978). This reflects the empirical findings that place-context-dependent recognition had not been found by means of the same contextual manipulations through which place-context-dependent recall had been found (Godden \& Baddeley, 1975, 1980; Smith et al., 1978). However, many subsequent experiments have reported significant place-context-dependent recognition (Canas \& Nelson, 1986; Emmerson, 1986; Smith, 1985, 1986). These findings fatally damaged the well-accepted notion that place-context-dependent effects appear in recall but not in recognition.

However, methodological issues weakened half of the experiments that found significant context-dependent recognition (i.e., Canas \& Nelson, 1986; Smith, 1986). Canas and Nelson manipulated place context between the laboratory and a telephone call at home. This created a confound of context matching and test place, because participants in the mismatched-context condition always received the recognition test by phone at home, whereas those in the matchedcontext condition always received the test at the laboratory. Smith (1986) manipulated levels of processing at encoding and found that shallow processing produced place-contextdependent recognition, but deeper processing did not. However, Smith and his colleagues failed to replicate these findings, and they concluded that shallow processing does not produce place-context-dependent recognition (Smith, Vela, \& Williamson, 1988). Thus, it is still uncertain whether recognition of meaningful words is reliably influenced by changes in place context.

The equivocal findings of context-dependent recognition of meaningful words in our review of the literature seem to contradict the results of Smith and Vela's (2001) metaanalysis, in which the size of the context-dependent effect was nearly the same in recognition as in recall. The conditions based on meaningful words that were included in the meta-analysis and in our review were almost the same. However, the conditions in the meta-analysis included data on context-dependent recognition not only of meaningful materials, but also of unfamiliar or meaningless materials. More importantly, the unfamiliar or meaningless materials revealed large effect sizes of context-dependent recognition. The large effect size of recognition in the meta-analysis may thus have been caused by the data from the unfamiliar or meaningless materials (e.g., Dalton, 1993; Krafka \& Penrod, 1985; Malpass \& Devine, 1981; Smith \& Vela, 1992) and from the problematic studies (e.g., Canas \& Nelson, 1986; Smith, 1986).

We propose that these equivocal findings may be made mutually understandable by assuming the following two trends. First of all, there is a clear trend toward the appearance of place-context-dependent recognition being influenced by the familiarity or meaningfulness of the materials. It has been repeatedly reported that the recognition of unfamiliar or meaningless materials, such as unfamiliar faces and nonwords, is influenced by changes in place context (e.g., Dalton, 1993; Malpass \& Devine, 1981; Russo, Ward, Geurts, \& Scheres, 1999). For instance, Dalton found place-context-dependent recognition of unfamiliar faces; Russo et al. successfully replicated the place-context-dependent recognition of unfamiliar faces, and further found context-dependent recognition with nonwords but not with meaningful words. Moreover, various eyewitness memory studies have reported placecontext-dependent recognition of the unfamiliar faces of culprits (e.g., Krafka \& Penrod, 1985; Malpass \& Devine, 1981; Smith \& Vela, 1992). Thus, these findings indicate a trend toward the probability of finding place-context-dependent recognition decreasing with increasing familiarity or meaningfulness of the materials.

Furthermore, previous findings of place-contextdependent recognition of words show a general tendency for the probability of finding place-context-dependent recognition of meaningful materials to decrease with increasing study time. More specifically, Smith and his colleagues used 2.5 and $3 \mathrm{~s}$ as the study times per item and found some significant effects in one study (Smith, 1985), but not in another (Smith et al., 1978). Godden and Baddeley (1980) found no effect with a 2-s study time per item when participants were given $4 \mathrm{~s}$ breathing time after every three items, such that the functional study time was $3.3 \mathrm{~s}$ per item. Russo et al. (1999) used a 5-s study time per item and found significant context-dependent recognition discrimination with nonwords, but no effect with words. Smith et al. (1988), who found no context-dependent effects in any processing-level conditions, used $10 \mathrm{~s}$ per item in their Experiments 1 and 2 and $5 \mathrm{~s}$ per item in Experiment 4. Although the study time in their Experiment 3 was uncertain, the time needed to complete each orienting task may have been longer than $3 \mathrm{~s}$.

We did not include Emmerson's (1986) results when considering the trend in study times because of a number of unusual manipulations in that study. First of all, Emmerson used quite unusual environments: on a boat and underwater at great depth $(20 \mathrm{~m})$. This manipulation may have covaried with changes in internal factors such as nervousness, anxiety, and relaxation. More importantly, Emmerson's manipulation may have covaried with the color of the slate on which the items were presented at both encoding and test; the board looked white on the boat, but possibly green deep underwater. About a decade later, the background color itself was found to produce context-dependent recognition (e.g., Isarida et al., 2005; Rutherford, 2004), regardless of the length of study time (Isarida et al., 2005). Furthermore, Emmerson used a far greater number of distractors than of targets - 16 targets and 84 distractors - whereas most experiments have used the 
same number of targets and distractors. Isarida (1992b) found that the appearance of place-context-dependent recognition decreased with an increase in the ratio of targets to distractors. In any case, it would be better for us to investigate the trend experimentally rather than through further review of previous studies.

In the present study, we examined the validity of the two trends we had identified in findings of place-contextdependent recognition. Experiment 1 tested whether the rate of place-context-dependent recognition of meaningful words would decrease with study time. More specifically, in Experiment 1 we used $1.5 \mathrm{~s}$ as the study time per item, which is shorter than the study times used in previous studies. Additionally, we included another condition with $4.0 \mathrm{~s}$ as the study time per item, because in previous experiments no significant context-dependent recognition of words has been found with study times longer than $3 \mathrm{~s}$ per item. Furthermore, in Experiment 2 we examined whether the meaningfulness of items influences place-contextdependent recognition. A trend for the effect size to decrease with greater familiarity and meaningfulness can be found in previous studies. In particular, Russo et al. (1999) found significant context-dependent recognition with nonwords but not with words. They compared the influences on words and nonwords within one study but in separate experiments, and used an incidental-learning paradigm. To test this trend more thoroughly, in Experiment 2 we manipulated word and nonword conditions within participants and used an intentional-learning paradigm. Since Experiment 2 had $4.0 \mathrm{~s} /$ item as the study time, no context-dependent recognition was predicted to be found with meaningful words.

Moreover, if these two trends were confirmed, we would further explore a possible account for the two trends. First of all, the encoding specificity principle alone cannot explain the trends. Although this principle can explain the contextdependent effects both in recognition discrimination and in simple recognition (hits and false alarms), it cannot specify the conditions under which context-dependent recognition should appear or not appear. Thus, we will discuss whether the encoding specificity principle can explain the trends by augmenting it with another principle, such as overshadowing or outshining.

"Overshadowing" refers to a situation in which the stronger of two cues presented during the encoding of items suppresses (overshadows) the weaker cue so as to associate with the presented items (e.g., Smith, 1994). Note that the respective cues would associate with the items if they were individually presented at encoding. Geiselman and Bjork (1980) found that voice-context-dependent recognition appeared for items that received primary rehearsal at encoding, but not for those that received secondary rehearsal. They explained their finding in terms of overshadowing. With secondary rehearsal, interitem associative cues formed that were more powerful than context cues, so that the interitem associative cues overshadowed the context cues. In contrast, with primary rehearsal, few interitem associative cues formed, so no overshadowing occurred. Their explanation is consistent with the meta-analysis of Smith and Vela (2001), which showed that associative processing decreases the size of the context-dependent effect.

On the other hand, according to the outshining principle, the stronger of two cues presented during the recognition test suppresses (outshines) the weaker cue to serve as retrieval cue (e.g., Smith, 1988, 1994). Smith (1988, 1994) proposed that an item-specific cue (a copy of the target item) is usually much stronger than a place-context cue. This is because each item-specific cue associates with only one target item, whereas a place context associates with all of the items, and the cue strength is assumed to be a reciprocal of the number of items associated with one cue (Watkins \& Watkins, 1975). The stronger, item-specific cues usually outshine the weaker, place-context cues, so that the place context cannot usually cue the target items. Otherwise, if the strength of the item-specific cues is weaker than the placecontext cues, the context will cue the items, and contextdependent recognition will hence appear.

The meaningfulness or familiarity of the materials (e.g., McGeoch, 1930; Underwood, 1966; Underwood \& Shultz, 1960) and study time (e.g., Glenberg, 1979) have been shown to determine the strengths of both interitem associations and item-specific cues. Thus, the principles of overshadowing and outshining provide similar predictions for context-dependent recognition-namely, that the size of context-dependent recognition will decrease with meaningfulness or familiarity and with study time. These similar predictions are consistent with the trends that we examined.

However, the overshadowing and outshining principles make different predictions with respect to context-dependent recall. Overshadowing predicts that strongly associated cues and weakly associated cues will be produced at encoding. If this were the case, then the different item-context associative strengths with meaningfulness and study time would produce different levels of free recall, as well as of recognition. Consequently, the overshadowing account — which consists of both the overshadowing and encoding specificity principles-predicts a decreasing size of context-dependent recall with increasing meaningfulness and study time. However, this prediction is contrary to the findings for free recall (Isarida, 1992a, 2005): The size of place-context-dependent effects in free recall was found to increase as a function of study time (Isarida, 2005) and of the number of cumulative rehearsals (Isarida, 1992a), even when interitem associative processing was not suppressed. This implies that the overshadowing account is not plausible.

In contrast, the outshining account - consisting of the outshining and encoding specificity principles-does not 
conflict with the findings of context-dependent effects in free recall (Isarida, 1992a, 2005), although this account does provide a prediction similar to that of the overshadowing account for context-dependent recognition. The outshining account focuses on the relative strengths of item-specific and place-context cues at the recognition test rather than on associative strengths formed at encoding. The item-specific cues are provided only during the recognition test, so the outshining account does not provide any prediction for context-dependent effects in free recall. Thus, the present study examined the validity of the outshining account for the findings of place-context-dependent recognition.

For the present study, we manipulated context by means of the combination of place and two more contextual elements, which remained stable throughout the encoding session, in order to reinstate the context defining a learning episode more completely. These two elements were the experimenter and the subsidiary task performed with the encoding task. Empirically, context manipulated by means of place and experimenter has been found to produce more reliable context-dependent effects than does a simple place manipulation (Smith \& Vela, 2001). Additionally, context manipulated by means of place and subsidiary task has been found to produce a significant context-dependent effect, even when place alone did not produce such an effect (Isarida \& Isarida, 2004). Isarida and colleagues have consistently found reliable context effects with combined manipulations of place and other elements, such as experimenter, subsidiary task, and background music (see, e.g., Isarida, 1992a, 2005; Isarida \& Isarida, 2006; Isarida \& Morii, 1986). Recently, this type of context, called "complex-place context," has been found to reinstate the context defining the original learning episode (Isarida \& Isarida, 2010). Therefore, in the present study we used complexplace context.

\section{Experiment 1}

Method

Participants The participants were 80 undergraduates enrolled in a Developmental Psychology course at Shizuoka Prefectural University in Shizuoka, Japan. They received extra course credit for their participation.

Design A $2 \times 2$ between-participants design was employed. The first factor was Context (same context [SC] vs. different context $[\mathrm{DC}])$, and the second factor was Study Time (1.5 vs. $4.0 \mathrm{~s}$ per item). The participants were randomly assigned to one of the $2 \times 2$ conditions, so that each group consisted of 20 participants. SC participants received the recognition test in the same context as at study, whereas DC participants received the recognition test in a context different from that of study.

Materials The stimuli were 80 unrelated two-kana Japanese words whose association values were more than 90 (Hayashi, 1976). The 80 items were randomly divided into two lists of 40 items across participants. One was used for a target list, and the other was used for a distractor list. Furthermore, the 40 tobe-remembered items were randomly divided into five subsets of eight items.

Context Context was manipulated by the combination of place, subsidiary task, and experimenter. Half of the SC participants were run in Place A with Experimenter A for both study and test, and the others were run in Place B with Experimenter B. All of the SC participants completed the same subsidiary task before testing that they had at study. On the other hand, half of the DC participants studied in Place A with Experimenter A and were tested in Place B with Experimenter B, and the others studied in Place B with Experimenter B and were tested in Place A with Experimenter A. None of the DC participants received any task before testing.

For the place element, the two places (A and B) were selected to be as perceptually distinct as possible, each differing from the other in size, illumination, objects, and apparatus. Place A was a $90 \times 90 \mathrm{~cm}$ corner of a room. In Place A, three gray panels were positioned to the front and sides of the participant, while the back remained open. The corner also contained a 17 -in. desktop computer screen, a mouse, a small table, and a chair. An experimenter gave instructions from behind the participant's back and then went behind the panel during the experimental session. Place B was a $550 \times 520 \mathrm{~cm}$ playroom for children. In Place $\mathrm{B}$, a big window overlooked buildings and trees, and many toys were scattered throughout the space. The experimenter and the participant sat across a $180 \times 180 \mathrm{~cm}$ table throughout the session.

A 10-min retention interval was used. During this interval, the participant engaged in a fragment-completion task with four-kanji compound words in Place N. Place N was a $380 \times 590 \mathrm{~cm}$ counseling room with two cabinets, a low table, two sofas, and six chairs. Places A, B, and $N$ were on the same floor of the same building of Shizuoka Prefectural University and were within a 1-min walk of each other.

For the task element, two tasks (A and B) were selected. Task A was a 30-s calculation task. A three-digit addition and/or subtraction problem was presented on the computer screen, and the participant was required to indicate the first digit of the solution by clicking with a mouse the corresponding numeric key presented on the computer screen as quickly and correctly as possible. The "Microsoft chime" tone was feedback for each correct response, and the 
"Microsoft chord" tone was feedback for each incorrect response. Regardless of correctness, each response set off the presentation of the next problem. Task B was a 30-s finemotor task in which the participant was required to carry beans with chopsticks one by one from one cup to a second cup as quickly as possible.

Either a male graduate student (Experimenter A) or a female graduate student (Experimenter B) served as the experimenter. Neither was acquainted with the participants.

Procedure The participants individually participated in two 15-min sessions separated by a 10-min retention interval. The procedure of the first session was identical for all of the conditions, except for the place, the task, and the experimenter. The participant came to a designated starting place and was then escorted by either Experimenter A or B to Place A or B. At the beginning of the first session, each participant received the following instructions:

The purpose of the present experiment is to investigate relationships between arithmetic or fine motor skill and mnemonic skill. More specifically, the experiment is designed to measure the changes in arithmetic or fine-motor performance before and after studying a lot of items. You must calculate (or carry beans) as fast and correctly as possible, and also must study as many words as possible in any order using any mnemonic strategies. At the end of the last calculation task, if double question marks (??) are presented, then your memory of the words will be tested, and if an end mark (END) is presented, then the first session is finished without testing.

The purpose of these instructions was to encourage the participants to study the words, but to keep their minds from thinking about the following test session, so that they would not rehearse the items during the retention interval. Following the instructions, the participant received five subsets of eight to-be-remembered items, presented one by one on a computer screen at a rate of 1.5 or $4.0 \mathrm{~s}$ per item (the interpresentation intervals were $0.5 \mathrm{~s}$ for both conditions). The presentation orders of the subsets and of the items within the subsets were randomized across participants. Each subset presentation was preceded and followed by a 30-s presentation of Task A or B. After the last Task A or B, every participant was shown the end mark, so that the study session was finished without testing.

After completion of the first session, the same experimenter escorted the participant to Place $\mathrm{N}$, where the participant engaged in an 8-min fragment-completion task with four-kanji compound words. The participant used the sofas and the table in that location. The experimenter left the participant in Place $\mathrm{N}$ after presenting the instructions for the fragment-completion task.
After $8 \mathrm{~min}$, the participant was escorted by the same experimenter to the same place (SC condition) or by the other experimenter to the other place (DC condition). Then the SC participants received a recognition test after completion of the same 30-s task used during the study session, whereas the DC participants received the recognition test after completion of the different 30-s task. The recognition test consisted of a sheet of A4 paper on which the target and distractor items were printed. The arrangement of the items was randomized across participants. The participant was required to mark a circle to the right of every target item that she or he recognized and an $\mathrm{X}$ to the right of every distractor item. Finally, the participant was debriefed.

Results and discussion

Table 1 shows hit rates, false alarm rates, and $d^{\prime}$ values as a function of Context (SC vs. DC) $\times$ Study Time (1.5 vs. $4.0 \mathrm{~s} /$ item). A series of $2 \times 2$ (Context $\times$ Study Time) analyses of variance (ANOVAs) for hit rates, false alarm rates, and $d^{\prime}$ were computed.

For hit rates, we found significant main effects of context $\left[F(1,76)=5.23, M S E=0.014, p<.05, \eta^{2}=.05\right]$ and of study time $\left[F(1,76)=23.71, M S E=0.014, p<.001, \eta^{2}=\right.$ $.22]$, and their interaction was significant $[F(1,76)=3.95$, $\left.M S E=0.014, p<.05, \eta^{2}=.04\right]$. Because the interaction was significant, further analyses were performed for each combination of the two factors. The results showed that the hit rate was significantly higher in the SC condition than in the DC condition for the 1.5 -s/item study time $[F(1,76)=9.18$, $\left.M S E=0.014, p<.01, \eta^{2}=.08\right]$, but not for the $4.0-\mathrm{s} /$ item study time $(F<1)$. The hit rate for the 4.0 -s/item study time was significantly higher than that for the 1.5-s/item study time in both the SC condition $[F(1,76)=4.12, M S E=$ $\left.0.014, p<.05, \eta^{2}=.04\right]$ and the DC condition $[F(1,76)=$ 23.57, MSE $\left.=0.014, p<.001, \eta^{2}=.22\right]$.

Table 1 Mean hit rates, false alarm rates, and $d^{\prime}$ as a function of context and study time in Experiment 1

\begin{tabular}{lllll}
\hline \multicolumn{3}{c}{$1.5 \mathrm{~s} /$ item } & & $4.0 \mathrm{~s} /$ item \\
\hline Context & Same & Different & Same & Different \\
Hit & & & & \\
$M$ & .723 & .613 & .796 & .788 \\
$S E$ & .023 & .028 & .026 & .026 \\
False Alarm & & & & \\
$M$ & .219 & .336 & .199 & .161 \\
$S E$ & .022 & .024 & .029 & .021 \\
$d^{\prime}$ & & & & \\
$M$ & 1.466 & 0.738 & 1.901 & 1.972 \\
$S E$ & .112 & .098 & .183 & .175 \\
\hline
\end{tabular}


For false alarm rates, the main effect of study time was significant $\left[F(1,76)=15.09, M S E=0.012, p<.001, \eta^{2}=.15\right]$, but the main effect of context was not significant $[F(1,76)=$ 2.67, $\left.M S E=0.012, p>.10, \eta^{2}=.03\right]$. The interaction was significant $\left[F(1,76)=10.04, M S E=0.012, p<.01, \eta^{2}=.10\right]$. Because the interaction was significant, further analyses were performed for each combination of the two factors. The results showed that the false alarm rate was significantly higher in the DC condition than in the SC condition for the 1.5-s/item study time $\left[F(1,76)=11.54, M S E=0.012, p<.01, \eta^{2}=.11\right]$, but not for the 4.0 -s/item study time $[F(1,76)=1.17, M S E=0.012$, $\left.p>.20, \eta^{2}=.01\right]$. The false alarm rate was significantly higher for the 1.5 -s/item study time than for the 4.0 -s/item study time in the DC condition $[F(1,76)=25.61, M S E=0.012, p<.001$, $\left.\eta^{2}=.24\right]$, but not in the SC condition $(F<1)$.

For $d^{\prime}$, the main effects of context $[F(1,76)=4.98$, $\left.M S E=0.432, p<.05, \eta^{2}=.04\right]$ and of study time $[F$ $\left.(1,76)=32.14, M S E=0.432, p<.001, \eta^{2}=.27\right]$ were significant, and their interaction was significant $[F(1,76)=$ 7.35, $\left.M S E=0.432, p<.01, \eta^{2}=.06\right]$. Because the interaction was significant, further analyses were performed for each combination of the two factors. The results showed that $d^{\prime}$ was significantly higher in the SC condition than in the DC condition for the 1.5 -s/item study time $[F(1,76)=29.69$, $\left.M S E=0.190, p<.001, \eta^{2}=.21\right]$, but not for the 4.0 -s/item study time $(F<1)$. Also, $d^{\prime}$ was significantly higher for the 4.0-s/item study time than for the 1.5-s/item study time in both the $\mathrm{SC}$ condition $[F(1,76)=4.12, M S E=0.014, p<.05$, $\left.\eta^{2}=.04\right]$ and the DC condition $[F(1,76)=35.12, M S E=$ $\left.0.432, p<.001, \eta^{2}=.29\right]$.

The present results show that the place-contextdependent effect in recognition discrimination was significant for the 1.5-s/item study time but not for the 4.0-s/item study time. Thus, the size of the place-context-dependent effect in recognition discrimination decreased with study time. The simple recognition hit rate and $d^{\prime}$ showed almost identical patterns of results. In contrast, the false alarm rate shows a reversed pattern from those for $d^{\prime}$ and the hit rate. For the 1.5-s/item study time, the false alarm rate was higher in the DC condition than in the SC condition, whereas $d^{\prime}$ and hit rate were higher in the SC than in the DC condition. However, this reversed effect was eliminated with a $4.0-\mathrm{s} /$ item study time.

\section{Experiment 2}

In Experiment 2, we examined whether the meaningfulness of the to-be-remembered material influences place-contextdependent recognition. As described in the introduction, the evidence for place-context-dependent recognition has been equivocal for meaningful materials, such as words, but clear for unfamiliar or meaningless materials, such as unfamiliar faces and nonwords. Experiment 2 was designed to more directly examine the influence of meaningfulness on placecontext-dependent recognition. Experiment 1 had shown that the reinstatement of place context did not produce context-dependent recognition of words in the 4.0 -s/item study-time condition. If place-context reinstatement were to produce context-dependent recognition of nonwords in the 4.0 -s/item condition, the meaningfulness of the to-beremembered materials would determine the occurrence of context-dependent recognition.

\section{Method}

Participants The participants were 40 undergraduates enrolled in a Developmental Psychology course at Shizuoka Prefectural University in Shizuoka, Japan. They had not participated in Experiment 1, and received extra course credit for their participation.

Design A $2 \times 2$ mixed-participants design was employed. The first factor was Context (SC vs. DC) as a betweenparticipants factor, and the second factor was Meaningfulness of Items (words vs. nonwords) as a within-participants factor. The participants were randomly assigned to either the SC or the DC condition, so that each condition consisted of 20 participants.

Materials The stimuli were 40 unrelated two-kana Japanese words whose association values were no less than 90 (Hayashi, 1976) and 40 two-letter Japanese nonwords whose association values were less than 50 (Hayashi, 1976). The 40 words were randomly divided into two lists of 20 words across participants, and the 40 nonwords were also divided into two lists of 20 words across participants. One word list and one nonword list were used as the target lists, and the other word and nonword lists were used as the distractor lists.

Context The context manipulation of Experiment 2 was identical to that of Experiment 1.

Procedure Each participant received four subsets of ten tobe-remembered items, presented one by one on a computer screen at a rate of $4.0 \mathrm{~s}$ per item (the interpresentation intervals were each $0.5 \mathrm{~s}$ ). Two of the four subsets consisted of words, and the other two consisted of nonwords. These assignments were randomized across participants. Otherwise, the procedure of Experiment 2 was identical to that of Experiment 1.

\section{Results}

Table 2 shows hit rates, false alarm rates, and $d^{\prime}$ values as a function of context (SC vs. DC) and meaningfulness (words 
Table 2 Mean hit rates, false alarm rates, and $d^{\prime}$ as a function of context and meaningfulness in Experiment 2

\begin{tabular}{lllll}
\hline & Words & \multicolumn{3}{l}{ Nonwords } \\
\hline Context & Same & Different & Same & Different \\
Hit & & & & \\
$M$ & .765 & .768 & .733 & .598 \\
$S E$ & .029 & .028 & .029 & .032 \\
False Alarm & & & & \\
$M$ & .338 & .343 & .340 & .443 \\
$S E$ & .021 & .020 & .021 & .023 \\
$d^{\prime}$ & & & & \\
$M$ & 1.252 & 1.241 & 1.126 & 0.441 \\
$S E$ & 0.586 & 0.456 & 0.455 & 0.463 \\
\hline
\end{tabular}

vs. nonwords). A series of $2 \times 2$ (Context $\times$ Meaningfulness) ANOVAs for hit rates, false alarm rates, and $d^{\prime}$ were computed.

For the hit rates, the main effects of context $[F(1,38)=$ 4.98, MSE $\left.=0.018, p<.05, \eta^{2}=.05\right]$ and meaningfulness $[F$ $\left.(1,38)=10.79, M S E=0.019, p<.05, \eta^{2}=.12\right]$ were significant, and their interaction was also significant $[F(1$, $\left.38)=4.97, M S E=0.019, p<.05, \eta^{2}=.05\right]$. Because the interaction was significant, further analyses were performed for each combination of the two factors. The results showed that the hit rate was significantly higher in the $\mathrm{SC}$ condition than in the DC condition for nonwords $[F(1,38)=9.59$ $\left.M S E=0.016, p<.01, \eta^{2}=.10\right]$, but not for words $(F<1)$. The hit rate for words was significantly higher than the hit rate for nonwords in the DC condition $[F(1,38)=15.21$, $\left.M S E=0.019, p<.001, \eta^{2}=.16\right]$, but not in the SC condition $(F<1)$.

For the false alarm rates, the main effects of context $[F(1$, 76) $\left.=6.07, M S E=0.010, p<.05, \eta^{2}=.07\right]$ and meaningfulness $\left[F(1,76)=5.56, M S E=0.009, p<.05, \eta^{2}=.05\right]$ were significant, and their interaction was also significant $[F$ $\left.(1,76)=5.03, M S E=0.009, p<.05, \eta^{2}=.05\right]$. Because the interaction was significant, further analyses were performed for each combination of the two factors. The results showed that the false alarm rate was significantly higher in the DC condition than in the $\mathrm{SC}$ condition for nonwords $[F(1,38)=$ 11.13, MSE $\left.=0.009, p<.01, \eta^{2}=.12\right]$, but not for words $(F<1)$. The false alarm rate for nonwords was significantly higher than that for words in the DC condition $[F(1,38)=$ $\left.10.59, M S E=0.009, p<.01, \eta^{2}=.11\right]$, but not in the SC condition $(F<1)$.

For $d^{\prime}$, the main effects of context $[F(1,38)=9.51$, $\left.M S E=0.254, p<.01, \eta^{2}=.09\right]$ and meaningfulness were significant $\left[F(1,38)=16.66, M S E=0.257, p<.001, \eta^{2}=.15\right]$, and their interaction was again significant $[F(1,38)=8.81$, $\left.M S E=0.257, p<.01, \eta^{2}=.08\right]$. Because the interaction was significant, further analyses were performed for each combination of the two factors. The results showed that $d^{\prime}$ was significantly higher in the SC condition than in the DC condition for nonwords $[F(1,38)=18.18, M S E=0.257, p<$ $\left..001, \eta^{2}=.16\right]$, but not for words $(F<1)$. Also, $d^{\prime}$ was significantly higher for words than for nonwords in the DC condition $\left[F(1,38)=24.85, M S E=0.257, p<.001, \eta^{2}=\right.$ $.225]$, but not in the SC condition $(F<1)$.

\section{Discussion}

In Experiment 2, the hit rate and $d^{\prime}$ showed almost identical patterns of results. The nonwords showed a significant context-dependent effect, but the words did not. The results for words in Experiment 2 were identical to those in the 4-s/ item study-time condition of Experiment 1. Also, these results were consistent with previous results. For instance, Russo et al. (1999) found that nonwords yielded a significant context-dependent effect in a 5-s/item study-time condition but that words did not. Furthermore, words produced significantly higher hit rates and $d^{\prime}$ than did nonwords in the $\mathrm{DC}$ condition, whereas there was no difference in hit rates or $d^{\prime}$ between words and nonwords in the SC condition. The null effect of meaningfulness in the SC condition may imply that the context cues may not have enhanced the hit rates for words.

The false alarm rate showed the reverse patterns of results, as compared to the hit rate and $d^{\prime}$. First, nonwords produced a significant reversal effect of context; that is, the DC participants showed a higher false alarm rate than did the SC participants. Second, in the DC condition, nonwords showed a higher false alarm rate than did words, suggesting that the context cue suppressed "yes" responses to new items (i.e., false alarms). Third, words produced neither a context-dependent effect nor a reversal effect, which was identical to the results found in the 4-s/item study-time conditions of Experiment 1.

\section{General discussion}

The present findings confirmed two types of trends that the appearance or the size of place-context-dependent effects in recognition discrimination decreases with both study time and the meaningfulness of the materials. More specifically, significant context-dependent effects in recognition discrimination of meaningful words were found in the $1.5-\mathrm{s} /$ item study-time conditions of Experiment 1. Smith and his colleagues used 2.5- and 3-s study times per item and found some significant effects (Smith, 1985; Smith et al., 1978). However, no context-dependent recognition was found when the study time was longer than $3 \mathrm{~s}$ per item (e.g., the 4.0-s/item study-time conditions of Exp. 1 and the word condition of Exp. 2; Godden \& Baddeley, 1980; Russo et 
al., 1999; Smith et al., 1988). On the other hand, significant context-dependent effects in recognition discrimination have been found with nonwords, even when the study times per item were $4.0 \mathrm{~s}$ (Exp. 2) and $5.0 \mathrm{~s}$ (Russo et al., 1999), when no context-dependent recognition of meaningful words was found. These results indicate that both study time and meaningfulness influence place-context-dependent recognition.

These trends are consistent with the predictions from the outshining account, which consists of the encoding specificity and outshining principles. The encoding specificity principle explains well the context-dependent effects both in recognition discrimination and in simple recognition (hits and false alarms). According to the encoding specificity principle, reinstating the study context will facilitate retrieval of the study episode, so that context-dependent effects in recognition discrimination will be found. Furthermore, the retrieved study episode will enhance discrimination between targets and distractors, because participants perceived and encoded the targets within the retrieved episode, but not the distractors. The enhanced discrimination between targets and distractors will then increase the hit rate and decrease the false alarm rate. As a result, a positive contextdependent effect on the hit rate and a reversal of this effect on the false alarm rate will be found. These predictions were clearly confirmed in the 1.5 -s/item study-time condition of Experiment 1 and the nonword condition of Experiment 2. Otherwise, if the item-specific cues outshine the placecontext cues, reinstating the context will not facilitate retrieval of the study episode. As a result, no contextdependent effect will be found in hit rates, false alarm rates, or recognition discrimination. In other words, any positive context-dependent effects on the hit rate will not be canceled out by the false alarm rate. These predictions were clearly confirmed in the 4.0-s/item study-time condition of Experiment 1 and the word condition of Experiment 2.

Moreover, the outshining principle specifies the conditions under which context-dependent recognition should appear or not appear, in terms of the relative strength of item-specific cues relative to context cues. The strength of item-specific cues has been found to be increased both by the meaningfulness of the materials (e.g., McGeoch, 1930; Underwood, 1966; Underwood \& Shultz, 1960) and by study time (e.g., Glenberg, 1979). Hence, the item-specific cues would be more likely to outshine the context cues as both the meaningfulness of the materials and the study time increased. Accordingly, the outshining principle predicts that the probability of context-dependent recognition will decrease both with study time and with the meaningfulness of the materials. This prediction is consistent with the trends confirmed by the present and previous studies.

It should be noted that the depth of processing of words is also recognized as determining the strength of item-specific cues (e.g., Craik, 1979; Craik \& Lockhart, 1972). Nonetheless, the depth of processing has been reported as not influencing place-context-dependent recognition (Smith et al., 1988). Smith et al. (1988) found no context-dependent recognition for either deeply processed or shallowly processed words in any of their four experiments. However, the findings of Smith et al. (1988) may be interpreted as indicating not only that the depth of processing does not influence context-dependent recognition, but also that potential influences of the depth of processing are suppressed by certain factors other than the level of processing. In fact, all of the experiments of Smith et al. (1988) may have been subject to the trends involving meaningfulness of the materials and study time, because all of their experiments used meaningful words and long ( $5 \mathrm{~s}$ or so per item) study times. Thus, it may be not plausible to conclude only on the basis of Smith et al. (1988) findings that the depth of processing does not influence context-dependent recognition.

In the present study, we found that the appearance of context-dependent recognition is influenced by the study time and the meaningfulness or familiarity of the materials. Study time and meaningfulness may not directly influence the appearance or size of context-dependent recognition, but rather the strength of item-specific cues may mediate the effects of study time or meaningfulness on contextdependent recognition. Both study time and meaningfulness have been found to increase the strength of item-specific cues (e.g., Glenberg, 1979; Underwood, 1966), and the cue strength may in turn influence the appearance or size of context-dependent recognition. Furthermore, the strength of context cues also influences the appearance or size of context-dependent recognition, and the context cue strength may be determined by the method of the manipulation used to reinstate context. For the present experiments, we used complex-place context (i.e., manipulating place, subsidiary task, and experimenter), which could have raised the hit levels more than would be found for simple-place contexts. As we discussed before, complex-place contexts can produce greater context-dependent effects than do simpleplace contexts (e.g., Isarida \& Isarida, 2004; Smith \& Vela, 2001).

The present results indicate that item-specific cues and context cues do not function additively, but rather compete with each other. More specifically, hit rates may reflect the stronger of the two cues. The hit rates under DC conditions reflect only the strength of item-specific cues, because only item-specific cues, and not context cues, are provided in the DC condition. On the other hand, the hit rates under SC conditions can reflect the strengths of both item-specific and context cues. In the present results, where the hit rate in the DC condition was about .60 or less, the context appeared to raise the $\mathrm{SC}$ hit rate to about .72 or more. In contrast, the context appeared to do nothing when the hit rate was about 
.75 or more, although there was still a latitude of .20 or more before reaching ceiling. This implies that item-specific cues and context cues do not function additively, but rather competitively. Furthermore, context may raise the hit rate to about .70 or so, regardless of the hit rates in the DC condition. Because context information is assumed to be accessed automatically (e.g., Glenberg, 1979; Murnane et al., 1999), context may always serve as a retrieval cue to remember the study episode. If item-specific cues produce a hit rate of only .60 or less, then context cues will compensate for the lower hit rate. Otherwise, if the item-specific cues produce a hit rate of .70 or higher, an influence of context cues will not appear. These processes have been called "outshining" (Smith, 1988, 1994).

The dual-process model explains recognition processes both by recollection and by familiarity judgments (see Yonelinas, 2002, for a review). The present results for place-context-dependent recognition can be explained in terms only of remembering of past episodes, which may be similar to the recollection processes proposed in the dualprocess model. In contrast, the findings from various visual contexts (e.g., Dougal \& Rotello, 1999; Hockley, 2008; Murnane \& Phelps, 1993, 1994, 1995; Murnane et al., 1999) other than the background-color context (e.g., Isarida et al., 2005; Rutherford, 2004) cannot be explained by recollection, but only by familiarity judgment processes, as proposed by the ICE theory (e.g., Murnane et al., 1999). Recollection and familiarity judgments make different predictions, especially regarding the false alarm rate as a function of context. As we described above, recollection of the study episode will increase the hit rate and decrease the false alarm rate, so that that the false alarm rate in the DC condition is predicted to be higher than that in SC condition. In contrast, according to a familiarity judgment, the false alarm rate will be enhanced by the familiar study (old) context, but not by the unfamiliar new context. Consequently, the false alarm rate in SC condition would be predicted to be higher than that in $\mathrm{DC}$ condition.

Several experiments have classified "old" responses in recognition as "remember" responses, which are assumed to reflect conscious recollection or remembering of past episodes, or as "know" responses, which are assumed to reflect familiarity judgments, and have found that "remember" responses revealed context-dependent recognition discrimination, but "know" responses did not (Gruppuso et al., 2007; Macken, 2002). This may imply that most visualcontext-dependent recognition processes also reflect conscious recollection (e.g., Gruppuso et al., 2007; Macken, 2002). However, further examining the details of the relevant conditions raises several questions about the dualprocess account of recognition (Hockley, 2008). In these studies, false alarm rates were almost always higher in the SC condition than in the DC condition. Even for "remember" responses, the false alarm rate was much higher in the SC than in the DC condition (Gruppuso et al., 2007; Macken, 2002). It should be noted that participants would remember nothing about distractors because of the study (old) context, because the distractors had not been presented, and hence had not been associated with the study context. Possibly, "remember" responses may reflect a certain subjective sense or feeling of remembering, but not veridical remembering of past episodes. Thus, the recognition processes depending on visual contexts other than a background-color context may reflect familiarity judgments rather than recollection.

Murnane et al. (1999) claimed that explanations relying on the principles of episode-defining context and encoding specificity may be flawed, because none of the explanations involving these two principles for the varying recognition findings over the past two decades (see Smith, 1994, for a review) have proven wholly satisfactory. Thus, Murnane and his colleagues proposed the ICE theory (Murnane et al., 1999), which is based on a global-matching view of context effects (Murnane \& Phelps, 1993, 1994, 1995). The ICE theory explains that recognition of a past episode involves familiarity judgment processes based on the global activation of item, context, and ensemble information. To demonstrate this theory, Murnane and his colleagues successfully demonstrated their theory using simple visual context and a background-picture context (Murnane \& Phelps, 1993, 1994, 1994; Murnane et al., 1999). Murnane and Phelps (1993) claimed that simple visual context meets Bjork and Richardson-Klavehn's (1989) definition of incidental context, in that visual contexts are independent of the focal information, should not influence the participants' cognition about the focal information, and are not predictive of correct responses at test. As such, most researchers may recognize that other types of visual context also meet the definition of incidental context.

Many researchers have followed Murnane and his colleagues' methodology (e.g., Dougal \& Rotello, 1999; Gruppuso et al., 2007; Hockley, 2008; Macken, 2002). Consequently, many studies on context-dependent recognition using various visual contexts (Dougal \& Rotello, 1999; Gruppuso et al., 2007; Hockley, 2008; Isarida et al., 2005; Macken, 2002; Murnane \& Phelps, 1993, 1994, 1995; Murnane et al., 1999; Rutherford, 2004) have been published since 1993, whereas only a few place-context studies have been done (Dalton, 1993; Russo et al., 1999). Although both place and visual contexts function as incidental environmental contexts, the present and previous results about place and visual contexts imply that they have different functions. Importantly, some issues related to place context are still to be clarified. Thus, studies of visual contexts alone are insufficient for clarifying the whole mechanism of environmental context-dependent recognition and episodic memory. Further studies on place context will be needed. 
Author note This research was supported in part by Grant-in-Aid for Scientific Research (C) 21530761 and (C) 18530580 from Japan Society for the Promotion of Science. Portions of this research were reported at the 55th Annual Meeting of the Japanese Psychological Association, Sendai, Japan; at the 73rd Annual Meeting of the Japanese Psychological Association, Kyoto, Japan. Correspondence should be addressed to Takeo Isarida, Faculty of Informatics, Shizuoka University, 3-5-1 Johoku, Hamamatsu, 432-8011 Japan (e-mail: isarida@inf.shizuoka.ac.jp).

\section{References}

Baddeley, A. D. (1982). Domains of recollection. Psychological Review, 89, 708-729. doi:10.1037/0033-295X.89.6.708

Bjork, R. A., \& Richardson-Klavehn, A. (1989). On the puzzling relationship between environmental context and human memory. In C. Izawa (Ed.), Current issues in cognitive processes: The Tulane Flowerre Symposium on cognition (pp. 313-344). Hillsdale: Erlbaum.

Canas, J. J., \& Nelson, D. L. (1986). Recognition and environmental context: The effect of testing by phone. Bulletin of the Psychonomic Society, 24, 407-409.

Craik, F. I. M. (1979). Human memory. Annual Review of Psychology, 30, 63-102.

Craik, F. I. M., \& Lockhart, R. S. (1972). Levels of processing: A framework for memory research. Journal of Verbal Learning and Verbal Behavior, 11, 671-684. doi:10.1016/S0022-5371 (72)80001-X

Dalton, P. (1993). The role of stimulus familiarity in context-dependent recognition. Memory \& Cognition, 21, 223-234.

Dougal, S., \& Rotello, C. M. (1999). Context effects in recognition memory. The American Journal of Psychology, 112, 277-295. doi: $10.2307 / 1423354$

Eich, E. (1995). Mood as a mediator of place dependent memory. Journal of Experimental Psychology. General, 124, 293-308. doi:10.1037/0096-3445.124.3.293

Emmerson, P. G. (1986). Effects of environmental context on recognition in an unusual environment. Perceptual and Motor Skills, 63, $1047-1050$.

Geiselman, R. E., \& Bjork, R. A. (1980). Primary versus secondary rehearsal in imagined voices: Differential effects on recognition. Cognitive Psychology, 12, 188-205.

Glenberg, A. M. (1979). Component-levels theory of the effects of spacing of repetitions on recall and recognition. Memory \& Cognition, 7, 95-112.

Godden, G., \& Baddeley, A. (1975). Context-dependent memory in two natural environments: On land and underwater. British Journal of Psychology, 6, 355-369.

Godden, G., \& Baddeley, A. (1980). When does context influence recognition memory? British Journal of Psychology, 71, 99104.

Gruppuso, V., Lindsay, D. S., \& Masson, M. E. J. (2007). I'd know that face anywhere! Psychonomic Bulletin \& Review, 14, 1085-1089. doi:10.3758/BF03193095

Hayashi, T. (1976). New norms of nonsense syllables. Tokyo: Tokai University Press.

Hockley, W. E. (2008). The effects of environmental context on recognition memory and claims of remembering. Journal of Experimental Psychology: Learning, Memory, and Cognition, 34, 1412-1429. doi:10.1037/a0013016

Isarida, T. (1992a). Influences of environmental-context changes on rehearsal effects in episodic memory. Japanese Journal of Psychology, 63, 262-268.
Isarida, T. (1992b, September). Influences of the number of alternatives on environmental context-dependent effects in forced-choice recognition. Paper presented at 56th Annual Meeting of Japanese Psychological Association, Kyoto, Japan.

Isarida, T. (2005). Study-time effect on free recall within and out of context. Memory, 13, 785-795.

Isarida, T., \& Isarida, T. K. (2004). Effects of environmental context manipulated by the combination of place and task on free recall. Memory, 12, 376-384.

Isarida, T., \& Isarida, T. K. (2006). Influences of environmental context on the recency effect in free recall. Memory \& Cognition, 34, 787-794.

Isarida, T., \& Isarida, T. K. (2010). Effects of simple- and complexplace contexts in the multiple-context paradigm. Quarterly Journal of Experimental Psychology, 63, 2399-2412.

Isarida, T., Isarida, T. K., \& Okamoto, K. (2005). Influences of cueoverload on background-color context effects in recognition. Japanese Journal of Cognitive Psychology, 3, 45-54.

Isarida, T., \& Morii, Y. (1986). Contextual dependence of the spacing effect in free recall. Japanese Journal of Psychology, 57, 20-26.

Krafka, C., \& Penrod, S. D. (1985). Reinstatement of context in a field experiment on eyewitness identification. Journal of Personality and Social Psychology, 49, 58-69.

Macken, W. J. (2002). Environmental context and recognition: The role of recollection and familiarity. Journal of Experimental Psychology: Learning, Memory, and Cognition, 28, 153-161. doi:10.1037/0278-7393.28.1.153

Malpass, R. S., \& Devine, P. G. (1981). Guided memory in eyewitness identification. Journal of Applied Psychology, 66, 343-350.

McGeoch, J. A. (1930). The influence of associative value upon the difficulty of nonsense-syllable. Journal of Genetic Psychology, 37, 421-426.

Murnane, K., \& Phelps, M. P. (1993). A global activation approach to the effect of changes in environmental context on recognition. Journal of Experimental Psychology: Learning, Memory, and Cognition, 19, 882-894. doi:10.1037/0278-7393.19.4.882

Murnane, K., \& Phelps, M. P. (1994). When does a different environmental context make a difference in recognition? A global activation model. Memory \& Cognition, 22, 584-590.

Murnane, K., \& Phelps, M. P. (1995). Effects of changes in relative cue strength on context-dependent recognition. Journal of Experimental Psychology: Learning, Memory, and Cognition, 21, 158-172. doi:10.1037/0278-7393.21.1.158

Murnane, K., Phelps, M. P., \& Malmberg, K. (1999). Contextdependent recognition memory: The ICE theory. Journal of Experimental Psychology. General, 128, 403-415. doi:10.1037/ 0096-3445.128.4.403

Russo, R., Ward, G., Geurts, H., \& Scheres, A. (1999). When unfamiliarity matters: Changing environmental context between study and test affects recognition memory for unfamiliar stimuli. Journal of Experimental Psychology: Learning, Memory, and Cognition, 25, 488-499. doi:10.1037/0278-7393.25.2.488

Rutherford, A. (2004). Environmental context-dependent recognition memory effects: An examination of ICE model and cue-overload hypotheses. Quarterly Journal of Experimental Psychology, 57, 107-127.

Smith, S. M. (1979). Remembering in and out of context. Journal of Experimental Psychology: Human Learning and Memory, 5, 460471. doi:10.1037/0278-7393.5.5.460

Smith, S. M. (1985). Environmental context and recognition memory reconsidered. Bulletin of the Psychonomic Society, 23, 173-176.

Smith, S. M. (1986). Environmental context-dependent recognition memory using a short-term memory task for input. Memory \& Cognition, 14, 347-354.

Smith, S. M. (1988). Environmental context-dependent memory. In G. M. Davis \& D. M. Thomson (Eds.), Memory in context: Context in memory (pp. 13-33). New York: Wiley. 
Smith, S. M. (1994). Theoretical principles of context-dependent memory. In P. Morris \& M. Gruenberg (Eds.), Theoretical aspects of memory (pp. 168-195). New York: Routledge.

Smith, S. M., Glenberg, A., \& Bjork, R. A. (1978). Environmental context and human memory. Memory \& Cognition, 6, 342-353. doi:10.3758/BF03197465

Smith, S. M., \& Vela, E. (1992). Environmental context-dependent eyewitness recognition. Applied Cognitive Psychology, 6, 125-139.

Smith, S. M., \& Vela, E. (2001). Environmental context-dependent memory: A review and meta-analysis. Psychonomic Bulletin \& Review, 8, 203-220. doi:10.3758/BF03196157

Smith, S. M., Vela, E., \& Williamson, J. E. (1988). Shallow input processing does not induce environmental context-dependent recognition. Bulletin of the Psychonomic Society, 26, 537-540.

Tulving, E. (1972). Episodic and semantic memory. In E. Tulving \& W. Donaldson (Eds.), Organization of memory (pp. 381-403). New York: Academic Press.
Tulving, E. (1983). Elements of episodic memory. New York: Oxford University Press.

Tulving, E., \& Thomson, D. M. (1973). Encoding specificity and retrieval processes in episodic memory. Psychological Review, 80, 352-373. doi:10.1037/h0020071

Underwood, B. J. (1966). Individual and group prediction of item difficulty for free learning. Journal of Experimental Psychology, 71, 673-679. doi:10.1037/h0023107

Underwood, B. J., \& Shultz, R. W. (1960). Meaningfulness and verbal learning. Philadelphia: Lippincott.

Watkins, O. C., \& Watkins, M. J. (1975). Buildup of proactive inhibition as a cue-overload effect. Journal of Experimental Psychology: Human Learning and Memory, 1, 442-452. doi:10.1037/ 0278-7393.1.4.442

Yonelinas, A. P. (2002). The nature of recollection and familiarity: A review of 30 years of research. Journal of Memory and Language, 46, 441-517. doi:10.1006/jmla.2002.2864 